\title{
Bacteriological Profile and the Antibiotic Susceptibility Pattern of Microorganisms Isolated from Pus/Wound Swab Isolates in Patients Attending a Tertiary Care Hospital in South India
}

\author{
S. Nithya Gomatheswari and T. Jeyamurugan* \\ Department of Microbiology, Thoothukudi Government Medical College, \\ Thoothukudi-628008, Tamil Nadu, India \\ *Corresponding author
}

\section{A B S T R A C T}

The aim of the study was to determine the commonly isolated aerobic microorganisms found in pus samples and their antibiotic sensitivity pattern. This study was conducted in the Government Medical College Hospital, Thoothukudi from January to December 2016. Pus samples received from various departments are processed by standard protocols. Antibiotic susceptibility was done by Kirby Bauer disc diffusion method. Among 1575 pus samples received for culture and sensitivity in the microbiology laboratory, $1126(71.49 \%)$

\section{Keywords}

Pus culture,

Sensitivity,

Resistance, MRSA,

ESBL.

Article Info

Accepted:

14 September 2017

Available Online:

10 October 2017 samples yielded positive culture and there was no growth in 449 (28.51\%) samples. Among the 1126 culture positive cases 665 (59.06\%) were male and 461 (40.94\%) were female and the male: female ratio is 1.44.Klebsiella species was predominantly isolated $253(22.5 \%)$ followed by Staphylococcus aureus was 208 (18.5\%), Escherichia coli was 194 (17.2\%), Pseudomonas aeruginosa was 178 (15.8\%), Proteus species was 166 (14.7\%), Citrobacter species was 33 (2.9\%), Acinetobacter species was 30(2.7\%), Coagulase Negative Staphylococcal species 55 (4.9\%), Enterococci species was 9 (0.8\%). Gram Negative bacteria were mostly susceptible to Amikacin, Piperacillin Tazobactum, Ceftazidime-clavulanic acid, Cefaperazone sulbactum and Imipenem whereas Gram positive organisms show $100 \%$ sensitivity to Vancomycin and linezolid. In this study, about $54 \%$ of the isolates were ESBL producers. The incidence of ESBL isolates were high in Escherichia coli $(60 \%)$ followed by Klebsiella species (56\%), Pseudomonas aeruginosa (51\%), Acinetobacter sp (50\%), Citrobacter species (52\%), Proteus species (49\%) among Gram Negative Bacilli. MRSA isolated were $41 \%$ of Staphylococcus aureus and $49 \%$ of Coagulase negative Staphylococcal species. The study gives the bacterial profile in the wound infections and its sensitivity pattern which is very important for clinicians to start empirical treatment for patients, while culture reports are awaited. This study suggests reserving carbapenem drugs for critically ill patients as the antibiotic pipeline is already dry.

\section{Introduction}

Pyogenic infections are associated with involvement of production of pus and bacteria. This may involve synergistic combination of aerobic and anaerobic species of bacteria which may be present either in singles or in mixed combinations which require antibiotic therapy. The probability of wound infections largely depends on local 
wound conditions, microbial burden and the host defense conditions. Effective treatment of wound infections depends upon proper understanding of causative pathogen, pathophysiology of the infectious process and pharmacology of the therapeutic agents. Multidrug resistant organisms continue to be important cause of Hospital acquired infection and pose a therapeutic challenge (Chaudhari et al., 2007; Gupta and Datta, 2007).

Because of irrational use of antibiotics, virulent strains adapt to the environment and it is a concern to the health care services. The antibiotic pipeline has become dry and it is the need of the hour to reserve antibiotics like carbapenems to multidrug resistant organisms (Amreliwala et al., 2015).

Continuous monitoring of bacterial profile from pus samples and its sensitivity would highlight variations in the resistance pattern of the organisms. The present study was done to monitor the aerobic bacterial profile and their antibiogram in pus samples in this region which is relevant for the clinicians in treating the patients empirically.

\section{Materials and Methods}

\section{Study design}

This study was conducted using a total number of 1575 pus/ wound samples received for aerobic culture and sensitivity from various Departments in the Microbiology laboratory of Government Medical College Hospital, Thoothukudi during a period from January 2016 to December 2016.

\section{Inclusion criteria}

All pus samples/ wound swab collected in sterile swabs and containers were included in the study.

\section{Exclusion criteria}

Pus samples received in unsterile containers were rejected.

\section{Identification and antibiotic susceptibility testing}

Pus samples were received in two sterile swab sticks or in sterile container. First swab stick is used for Gram staining and second one is used for culture. Received pus samples were processed on blood agar, MacConkey agar, Nutrient agar media and incubated at $37^{\circ} \mathrm{C}$ under aerobic condition and the organisms are identified by Gram stain, motility testing, biochemical reactions (Catalase, Oxidase, Indole, citrate utilisation, urease test, Triple sugar Iron test, Mannitol salt agar) using standard microbiological methods (Collee et al., 1996; Koneman, 2006). For antibiotic sensitivity, bacterial suspensions of the isolate is prepared and matched with 0.5 McFarland standard for lawn culture. The antimicrobial susceptibility testing were done by modified Kirby Bauer disc diffusion method and interpreted by CLSI guidelines 2017.Standard antibiotics like penicillin G (10units), amoxicillin-clavulanicacid (20/10 mcg), amikacin (30mcg), Gentamicin (10mcg), Tobramycin (10mcg), Erythromycin (15mcg), ciprofloxacin (5mcg), ceftriaxone (30 mcg), ceftazidime $(30 \mathrm{mcg})$, ceftazidime clavulanic acid (30/10mcg), Cefaperazone sulbactum (75/10mcg), cotrimoxazole $(1.25 / 23.75 \mathrm{mcg})$, piperacillin/tazobactum (100/10mcg), Vancomycin (30mcg), Linezolid (30 mcg) and cefoxitin (30mcg) for screening MRSA were obtained from Himedia, Mumbai India.

\section{Screening of MRSA (Methicillin resistant Staphylococcus aureus)}

Cefoxitin $30 \mu \mathrm{g}$ disc is considered as surrogate marker for detection of methicillin resistant Staphylococcus aureus isolates. Zone 
of inhibition of less than $21 \mathrm{~mm}$ is considered as Methicillin resistant (CLSI guidelines 2017).

\section{Screening of ESBL (Extended Spectrum Beta Lactamase)}

A disc of cefotaxime $(30 \mu \mathrm{g})$ and ceftazidime $(30 \mu \mathrm{g})$ were kept placed on the agar surface in addition to the other antimicrobial agents. The plate was incubated at $37^{\circ} \mathrm{C}$ overnight. The zone diameter of $<=27 \mathrm{~mm}$ and $<=22 \mathrm{~mm}$ for Cefotaxime and Ceftazidime respectively indicated that the isolate may produce ESBL.

\section{Phenotypic ESBL confirmatory method}

By using the phenotypic disc diffusion test (CDDT), all the organisms were screened for ESBL production. Organism was considered as ESBL producer, if the ceftazidime clavulanic acid zone size is more than or equal to $5 \mathrm{~mm}$ with comparison to ceftazidime disc zone size (CLSI guidelines 2017).

\section{Quality control}

Staphylococcus aureus ATCC 25923, Pseudomonas aeruginosa ATCC 27853 and E. coli ATCC 25922 were used as quality controls strains. Results obtained were analysed by percentages using MS Excel 2010.

\section{Results and Discussion}

Out of 1575 pus samples received for culture and sensitivity in the microbiology laboratory, 1126 (71.49\%) samples yielded positive culture and there was no growth in 449 (28.51\%) samples (Figure 1). Among the 1126 culture positive samples, 900 yielded pure bacterial isolates and 226 yielded mixed infection. Among the 1126 culture positive cases $665(59.06 \%)$ were male and 471
(41.8\%) were female and the male: female ratio is 1.44 (Table 1 ).

Among the 1126 culture positive samples, Klebsiella species was predominant bacterial isolate $253(22.5 \%)$ followed by Staphylococcus aureus was 208 (18.5\%), Escherichia coli was $194 \quad(17.2 \%)$, Pseudomonas aeruginosa was 178 (15.8\%), Proteus species was 166 (14.7\%), Citrobacter species was 33 (2.9\%), Acinetobacter species was 30 (2.7\%), Coagulase Negative Staphylococcus species was 55 (4.9\%), Enterococci species was 9 (0.8\%).

Polymicrobial infection with two or more organisms occurred in $20.1 \%$ cases. Staphylococcus aureus was most commonly isolated among the Gram positive cocci. Klebsiella $s p$ was most commonly isolated among Enterobacteriaceae followed by $E$. coli, Proteus sp. (Figure 2) (Table 2).

\section{Antibiotic susceptibility pattern of Organisms isolated}

Klebsiella species isolated were $68 \%$ sensitive to amikacin, $48 \%$ sensitive to Ceftazidime, $44 \%$ sensitive to Cetriaxone, $42 \%$ sensitive to Ceftazidime-clavulanic acid, $56 \%$ sensitive to Cefaperazone sulbactum and $83 \%$ sensitive to Imipenem. Antibiotic resistance of Klebsiella species is very high in third generation cephalosporins (Table 3 ).

E. coli isolated were susceptible to species isolated were $75 \%$ sensitive to amikacin, $44 \%$ sensitive to Ceftazidime, $40 \%$ sensitive to Cetriaxone, $64 \%$ sensitive to Cefaperazone sulbactum $56 \%$ sensitive to Ceftazidimeclavulanic acid and $89 \%$ sensitive to Imipenem (Table 3).

Proteus species isolated were susceptible to species isolated were $49 \%$ sensitive to amikacin, $31 \%$ sensitive to Ceftazidime, $51 \%$ 
sensitive to Cetriaxone, $72 \%$ sensitive to Cefaperazone sulbactum, $67 \%$ sensitive to Ceftazidime-clavulanic acid and $80 \%$ sensitive to Imipenem (Table 3).

Pseudomonas aeruginosa is sensitive to $74 \%$ sensitive to amikacin, $62 \%$ sensitive to Cefaperazone sulbactum, $79 \%$ sensitive to Piperacillin tazobactum, $44 \%$ sensitive to Ceftazidime, $40 \%$ sensitive to Cetriaxone, $47 \%$ sensitive to Ciprofloxacin, $54 \%$ sensitive to Ceftazidime-clavulanic acid and $87 \%$ sensitive to Imipenem (Table 4).

Acinetobacter $s p$ is sensitive to $83 \%$ sensitive to amikacin, $60 \%$ sensitive to Cefaperazone sulbactum, $73 \%$ sensitive to Piperacillin tazobactum, $50 \%$ sensitive to Ceftazidime, $47 \%$ sensitive to Cetriaxone, $53 \%$ sensitive to Ciprofloxacin, $57 \%$ sensitive to Ceftazidimeclavulanic acid and $87 \%$ sensitive to Imipenem (Table 4).

In this study, about $54 \%$ of the isolates were ESBL producers. The incidence of ESBL isolates were high in Escherichia coli $(60 \%)$ followed by Klebsiella species (56\%), Citrobacter species (52\%), Proteus species (49\%) among Enterobacteriaceae. Among non-fermenting gram negative bacilli ESBL was detected in Pseudomonas aeruginosa (51\%) followed by Acinetobacter species $(50 \%)$ (Table 6).

Fig.1 Pie chart showing culture positivity among pus sample

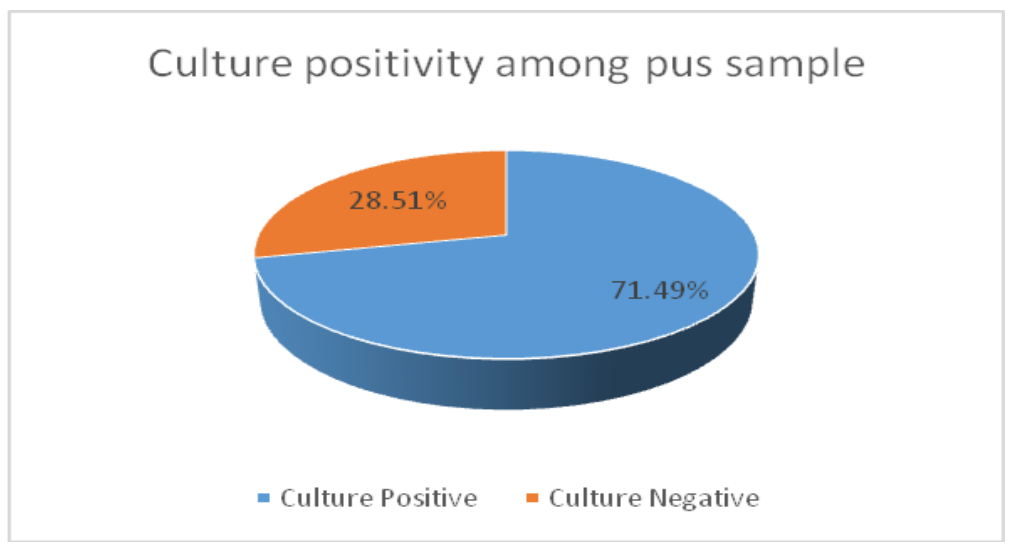

Fig.2 Bar Graph showing distribution of organisms isolated from pus samples

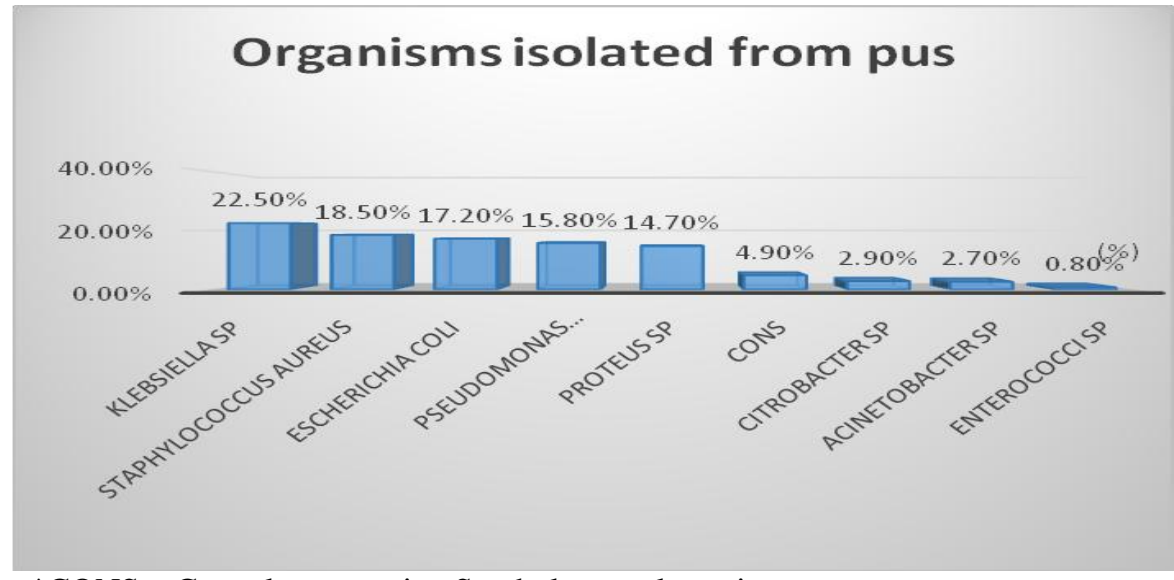

*CONS - Coagulase negative Staphylococcal species 
Table.1 Sex wise distribution of culture positive pus samples

\begin{tabular}{|l|l|}
\hline Sex & Culture positive $(\mathrm{n}=1126)$ \\
\hline Male & $665(59.06 \%)$ \\
\hline Female & $461(40.94 \%)$ \\
\hline
\end{tabular}

Table.2 Aerobic bacteria $(\mathrm{n}=1126)$ isolated from pus samples

\begin{tabular}{|l|l|l|}
\hline S.No. & Organism & Number $(\%)$ \\
\hline 1. & Klebsiella sp & $253(22.5 \%)$ \\
\hline 2. & Staphylococcus aureus & $208(18.5 \%)$ \\
\hline 3. & Escherichia coli & $194(17.2 \%)$ \\
\hline 4. & Pseudomonas aeruginosa & $178(15.8 \%)$ \\
\hline 5. & Proteus sp & $166(14.7 \%)$ \\
\hline 6. & CONS & $55(4.9 \%)$ \\
\hline 7. & Citrobacter sp & $33(2.9 \%)$ \\
\hline 8. & Acinetobacter sp & $30(2.7 \%)$, \\
\hline 9. & Enterococci sp & $9(0.8 \%)$ \\
\hline Total & & 1126 \\
\hline
\end{tabular}

Table.3 Pattern of antibiotic sensitivity and resistance for Enterobacteriaceae

\begin{tabular}{|l|l|l|l|l|l|l|l|l|}
\hline \multirow{2}{*}{$\begin{array}{l}\text { Name of } \\
\text { Antibiotics }\end{array}$} & \multicolumn{2}{|l|}{ Klebsiella $\mathrm{sp}(\mathrm{n}=253)$} & \multicolumn{2}{l|}{ E. coli $(\mathrm{n}=194)$} & \multicolumn{2}{l|}{ Proteus $\mathrm{sp}(\mathrm{n}=166)$} & \multicolumn{2}{l|}{ Citrobacter $\mathrm{sp}(\mathrm{n}=33)$} \\
\cline { 2 - 9 } & Sensitive & Resistant & Sensitive & Resistant & Sensitive & Resistant & Sensitive & Resistant \\
\hline Ampicillin & $42(17 \%)$ & $211(83 \%)$ & $47(24 \%)$ & $147(76 \%)$ & $52(31 \%)$ & $114(69 \%)$ & $12(36 \%)$ & $21(64 \%)$ \\
\hline Amikacin & $172(68 \%)$ & $81(32 \%)$ & $146(75 \%)$ & $48(25 \%)$ & $82(49 \%)$ & $84(51 \%)$ & $24(72 \%)$ & $6(18 \%)$ \\
\hline Gentamycin & $133(52 \%)$ & $120(48 \%)$ & $60(31 \%)$ & $134(69 \%)$ & $23(14 \%)$ & $143(86 \%)$ & $22(67 \%)$ & $8(24 \%)$ \\
\hline Ciprofloxacin & $110(43 \%)$ & $143(57 \%)$ & $98(51 \%)$ & $96(49 \%)$ & $34(20 \%)$ & $132(80 \%)$ & $17(51 \%)$ & $16(49 \%)$ \\
\hline Ceftazidime & $123(48 \%)$ & $130(52 \%)$ & $86(44 \%)$ & $108(56 \%)$ & $52(31 \%)$ & $114(69 \%)$ & $15(45 \%)$ & $18(55 \%)$ \\
\hline Ceftriaxone & $112(44 \%)$ & $141(56 \%)$ & $77(40 \%)$ & $117(60 \%)$ & $85(51 \%)$ & $81(49 \%)$ & $16(48 \%)$ & $17(52 \%)$ \\
\hline $\begin{array}{l}\text { Ceftazidime- } \\
\text { clavulanic acid }\end{array}$ & $105(42 \%)$ & $148(58 \%)$ & $108(56 \%)$ & $86(44 \%)$ & $112(67 \%)$ & $54(33 \%)$ & $11(33 \%)$ & $22(66 \%)$ \\
\hline $\begin{array}{l}\text { Cefaperazone } \\
\text { sulbactum }\end{array}$ & $142(56 \%)$ & $111(44 \%)$ & $124(64 \%)$ & $70(36 \%)$ & $120(72 \%)$ & $46(28 \%)$ & $19(58 \%)$ & $14(42 \%)$ \\
\hline Imipenem & $209(83 \%)$ & $44(17 \%)$ & $172(89 \%)$ & $22(11 \%)$ & $133(80 \%)$ & $33(20 \%)$ & $26(79 \%)$ & $7(21 \%)$ \\
\hline
\end{tabular}

Table.4 Pattern of antibiotic sensitivity and resistance for Non fermenters

\begin{tabular}{|c|c|c|c|c|}
\hline \multirow[t]{2}{*}{ Name of Antibiotics } & \multicolumn{2}{|c|}{ Pseudomonas aeruginosa $(\mathrm{n}=178)$} & \multicolumn{2}{|c|}{ Acinetobacter $\operatorname{spp}(\mathrm{n}=30)$} \\
\hline & Sensitive & Resistant & Sensitive & Resistant \\
\hline Ampicillin & $32(18 \%)$ & $146(82 \%)$ & $5(17 \%)$ & $25(83 \%)$ \\
\hline Amikacin & $131(74 \%)$ & $47(26 \%)$ & $25(83 \%)$ & $5(17 \%)$ \\
\hline Gentamicin & $69(39 \%)$ & $109(61 \%)$ & $17(57 \%)$ & $13(43 \%)$ \\
\hline Tobramycin & $138(78 \%)$ & $40(22 \%)$ & $25(83 \%)$ & $5(17 \%)$ \\
\hline Ciprofloxacin & $83(47 \%)$ & $95(53 \%)$ & $16(53 \%)$ & $14(47 \%)$ \\
\hline Cotrimoxazole & 0 & 0 & 0 & 0 \\
\hline Ceftazidime & $88(49 \%)$ & $90(51 \%)$ & $15(50 \%)$ & $15(50 \%)$ \\
\hline Ceftriaxone & $72(40 \%)$ & $106(60 \%)$ & $14(47 \%)$ & $16(53 \%)$ \\
\hline Ceftazidime-clavulanic acid & $96(54 \%)$ & $82(46 \%)$ & $17(57 \%)$ & $13(43 \%)$ \\
\hline Cefaperazone sulbactum & $110(62 \%)$ & $68(38 \%)$ & $18(60 \%)$ & $12(40 \%)$ \\
\hline Piperacillin-Tazobactum & $141(79 \%)$ & $37(21 \%)$ & $22(73 \%)$ & $8(27 \%)$ \\
\hline Imipenem & $155(87 \%)$ & $23(13 \%)$ & $26(87 \%)$ & $4(13 \%)$ \\
\hline
\end{tabular}


Table.5 Pattern of antibiotic sensitivity and resistance for Gram positive cocci

\begin{tabular}{|l|l|l|l|l|l|l|}
\hline \multirow{2}{*}{$\begin{array}{l}\text { Name of } \\
\text { Antibiotics }\end{array}$} & \multicolumn{2}{|l|}{$\begin{array}{l}\text { Staphylococcus aureus } \\
(\mathrm{n}=208)\end{array}$} & \multicolumn{2}{l|}{$\begin{array}{l}\text { CONS } \\
(\mathrm{n}=55)\end{array}$} & \multicolumn{2}{l|}{$\begin{array}{l}\text { Enterococci } \\
(\mathrm{n}=9)\end{array}$} \\
\cline { 2 - 7 } & Sensitive & Resistant & Sensitive & Resistant & Sensitive & Resistant \\
\hline Ampicillin & $48(26 \%)$ & $140(75 \%)$ & $18(33 \%)$ & $37(67 \%)$ & $3(33 \%)$ & $6(67 \%)$ \\
\hline Clindamycin & $113(60 \%)$ & $75(40 \%)$ & $14(25 \%)$ & $41(75 \%)$ & 0 & 0 \\
\hline Ciprofloxacin & $35(19 \%)$ & $153(81 \%)$ & $18(33 \%)$ & $37(67 \%)$ & 0 & 0 \\
\hline Erythromycin & $94(50 \%)$ & $94(50 \%)$ & $34(62 \%)$ & $21(38 \%)$ & $2(22 \%)$ & $7(78 \%)$ \\
\hline Amikacin & $124(66 \%))$ & $64(34 \%)$ & $40(73 \%)$ & $15(27 \%)$ & 0 & 0 \\
\hline Gentamycin(HLG) & 0 & 0 & 0 & 0 & $7(78 \%)$ & $2(22 \%)$ \\
\hline Cotrimoxazole & $62(33 \%)$ & $126(67 \%)$ & $13(24 \%)$ & $42(76 \%)$ & 0 & 0 \\
\hline Cefoxitin & $124(66 \%)$ & $64(34 \%)$ & $28(51 \%)$ & $27(49 \%)$ & 0 & 0 \\
\hline $\begin{array}{l}\text { Amoxicillin-clavulanic } \\
\text { acid }\end{array}$ & $76(41 \%)$ & $112(59 \%)$ & $30(54 \%)$ & $25(46 \%)$ & 0 & 0 \\
\hline Vancomycin & $208(100 \%)$ & 0 & $55(100 \%)$ & 0 & $9(100 \%)$ & 0 \\
\hline Linezolid & $208(100 \%)$ & 0 & $55(100 \%)$ & 0 & $9(100 \%)$ & 0 \\
\hline
\end{tabular}

Table.6 ESBL isolates in Gram negative bacilli

\begin{tabular}{|l|l|l|l|}
\hline Organisms & ESBL n (\%) & Non ESBL n (\%) & Total \\
\hline Klebsiella sp & $141(56 \%)$ & $112(44 \%)$ & 253 \\
\hline E. coli & $117(60 \%)$ & $77(40 \%)$ & 194 \\
\hline Proteus sp & $81(49 \%)$ & $85(51 \%)$ & 166 \\
\hline Citrobacter sp & $17(52 \%)$ & $16(48 \%)$ & 33 \\
\hline Pseudomonas sp & $90(51 \%)$ & $88(49 \%)$ & 178 \\
\hline Acinetobacter & $15(50 \%)$ & $15(50 \%)$ & 30 \\
\hline Total & $461(54 \%)$ & $393(46 \%)$ & 854 \\
\hline
\end{tabular}

Gram positive organisms mainly Staphylococcus aureus isolated were $100 \%$ sensitive to Vancomycin and Linezolid, 66\% sensitive to amikacin, $41 \%$ sensitive amoxicillin-clavulanic acid, $50 \%$ sensitive to Erythromycin and $60 \%$ sensitive to clindamycin. Methicillin resistant Staphylococcus aureus isolates were detected by cefoxitin disc diffusion method. $41 \%$ of Staphylococcus aureus and $49 \%$ of Coagulase negative Staphylococcus species were resistant to Cefoxitin (Table 5).

This study shows male preponderance $(59.06 \%)$ as compared to female $(41.80 \%)$. It closely corroborates with the study by Raghav Rao et al., (2014) which shows highest occurrence in males $(58.82 \%)$. This was similar to studies of siddiqui et al., (2002), Bhatt and Vassikar (2010), Pappu et al., (2011), Rajeshwar Rao et al., (2014).

Gram Negative bacteria were most predominantly isolated from pus culture samples compared to Gram Positive bacteria. This was similar to studies by Aziza zafar (2008), Ghosh et al., (2009), Zubair etal., (2011), Sarath babu et al.,(2012), Rajeswar Rao et al.,(2014), Ravichitra et al., (2014), Nirmala and Rajesh (2017).

Klebsiella $s p$ was the most commonly isolated organisms $253(23 \%)$. This was observed in other studies like Sarath babu et al., (2012), Rajeswar Rao et al., (2014), Kritu Panta et al., (2013), Ravichitra et al., (2014), Vijeta et al., 
(2015) and Nirmala and Rajesh (2017). In contrast, some studies Karia et al., (2013) highlighted Staphylococcus aureus was isolated in increased frequency whereas in other study by Ramesh Kannan et al., (2014) $E$. coli was isolated more commonly.

In this study, about $54 \%$ of the isolates were ESBL producers. While in India, the ESBL prevalence ranges from 60 to $70 \%$ (Menon et al., 2007) (Dalela, 2012). There is high incidence of ESBL isolates in Escherichia coli(60\%)followed by Klebsiella species (56\%), Citrobacter species (52\%),Proteus species (49\%) among gram negative bacilli under family Enterobacteriaceae similar to study of Basavaraj et al., (2011).

In this study $41 \%$ of Methicillin resistant Staphylococcus aureus is isolated which correlates with a study by Arora et al., (2010) from North India where the prevalence of MRSA was $46 \%$. In a study conducted by Indian network for Surveillance of Antimicrobial Resistance (INSAR) group, India shows the overall MRSA prevalence was 42 per cent in 2008 and 40 per cent in 2009 (INSAR group, India 2013).

The organisms most commonly isolated from the pus culture samples were Klebsiella species followed by Staphylococcus aureus and Pseudomonas aeruginosa. MRSA screening by cefoxitin disc diffusion method reveals $41 \%$ of Staphylococcus aureus and $49 \%$ of Coagulase negative Staphylococcus species. Among Gram Negative bacteria's, $54 \%$ of the isolates were ESBL producers. Among Gram Negative bacteria, all the organisms were highly sensitive to Aminoglycosides, Cefaperazone sulbactum, Piperacillin Tazobactum, Imipenem and Gram Positive bacteria's were all $100 \%$ sensitive to Vancomycin and Linezolid. This study did not address the detection of Amp C prevalence in our set up which remains to be the limitation and gives a scope to focus in the future research.

This study gives an outline of antibiotic susceptibility of clinical isolates which will help in formulating the local antibiotic policy for the hospital. This study suggests that beta lactum and its betalactamase combination like Piperacillin tazobactum or Cefaperazone sulbactum and aminoglycosides like Amikacin instead of choosing carbapenemsin treating hemodynamically stable patients. Hence this study would help in adhering to appropriate empirical therapy for the specific organisms and also prevent emergence and spread of resistant isolates.

Due to non-availability of newer antibiotics for the treatment purpose it is necessary to monitor resistance pattern among the clinical samples so that right choice of drug can reduce the burden to physicians during the patient care. This study emphasis to reserve carbapenems for only seriously ill patients.

\section{References}

Aizza Zafar, Naeema Anwar and Hasanejaz. Bacteriology of infected wounds $-\mathrm{A}$ study Conducted at Children's Hospital Lahore. Biomedica. 2008; 24:71-74.

Amreliwala, S., S. Durgad and A.Poojary Carbapenem sparing options for the treatment of ESBL and AmpC producing Enterobacteriaceae in hemodynamically stable patients an in vitro study. Int.J.Curr.Microbiol.App. Sci., (2015) 4(2): 513-521

Arora S, Devi P, Arora u, Devi B. Prevalence of Methicillin-resistant Staphylococcus aureus (MRSA) in a tertiary care hospital in northern India. J Lab Physicians 2010; 2: 78-81

BN Chaudhari, CRodrigues, V Balaji, R Iyer, U Sekar, C Wattal, DS Chitnis, TN Dhole, $\mathrm{S}$ Joshi. Incidence of ESBL 
producers amongst gram negative bacilli isolated from Intra-abdominal infections across India (Based on smart study 2007 Data) Poster GN 103 presented at IsAAR 2009.

Clinical Laboratory Standards Institute Performance Standards for antimicrobial susceptibility testing $\mathrm{M}$ 100, 27th edition, January 2017.

Colle. J. G., Digcid J.P, Fraser A.G. Mackie and MacCartny, Practical Medical Microbiology. $14^{\text {th }}$ edition: 1996; 413418.

D.V.M.V.S.V. Raghav Rao, Ranjan Basu, Debika Roy Biswas. Aerobic Bacterial Profile and Antimicrobial Susceptibility Pattern of Pus Isolates in a South Indian Tertiary Care Hospital. Journal of Dental and Medical Sciences Volume 13, Issue 3 Ver. II. (Mar. 2014), PP 59 62.

Dalela G., Prevalence of Extended Spectrum Beta Lactamase (ESBL) Producers among Gram Negative Bacilli from Various Clinical Isolates in a Tertiary Care Hospital at Jhalawar, Rajasthan, India. J Clin Diagn Res. 2012; Vol-6(2): 182-187.

Farah Siddiqi, Madahihah-bint-E-Masood, Noor-Us-Saba, Abdus Samad, Mazhar Qayyum and Ali Abbas Qazilbash. Antibiogram Sensitivity Pattern of Methicillin Resistant S. aureus isolates from Pus Sample. Pakistan Journal of Biological Science. 2002; 5(4): 491493.

Ghosh A, Karmakar PS, Pal J, Chakraborty N, Debnath NB, Mukherjee JD. Bacterial incidence and antibiotic sensitivity pattern in moderate and severe infections in hospitalized patients. J Indian Med Assoc. 2009, 107(1):21-2, 24-5.

Gupta V, Datta P. Extended-Spectrum betalactamases (ESBL) in community isolates from North India: frequency and predisposing factors. Int $\mathbf{J}$ Infect Dis., 2007. 11(1): 88.

Indian network for Surveillance of Antimicrobial Resistance (INSAR) group, India. Methicillin resistant Staphylococcus aureus (MRSA) in India: Prevalence and susceptibility pattern. Indian J Med Res 137, February 2013, pp 363-369.

Karia JB, Gadekar HB, Lakhani SJ. Study of bacterial profile of pus culture in Dhiraj General hospital. Indian J Surg Oncol. 2013;4(2):172-218.

Koneman's color atlas and textbook of diagnostic microbiology, 6th ed (pp 624-62). Philadelphia, PA: Lippincott Williams and Wilkins, 2006b.

Kritu Panta, Prakash Ghimire, Shiba Kumar Rai, Reena Kiran Mukhiya, Ram Nath Singh, Ganesh Rai. Antibiogram Typing of Gram Negative Isolates in Different Clinical Samples of a Tertiary Hospital. Asian Journal of Pharmaceutical and Clinical Research. 2013; 6:153-156.

Menon T, Bindu D, Kumar C P G, SNalini, Thirunarayan $\mathrm{M}$ A.Comparison of double disc and three dimensional methods to screen for ESBL producers in a tertiary care hospital. Indian Journal of Medical Microbiology 2006; 24:117120.

Nirmala sukumar, Rajesh Sengodan. Aerobic bacterial isolates and their susceptibility pattern from pus samples in a tertiary care Hospital in Tamilnadu. Int. J curr. Microbiological Applied sciences (2017) 6(6): 423-442.

Pappu AK, Sinha A, Johnson A. Microbiological profile of Diabetic foot ulcer. Calicut Medical Journal, 2011; 9(3) e2.

Rajeshwar Rao, S., L. Jaya Lakshmi1, S.Pavani, Vijendra Kawle and S. Jaya Prakash. Bacteriological Profile, Antibiogram of Burn wound Isolates and Detection of MRSA and ESBL 
Production at Tertiary Care Hospital, Hyderabad. World Journal of Pharmacy and Pharmaceuticals Sciences. 2014; 3: 1691-1698.

Rameshkannan S, Nileshraj G, Rameshprabu S, Mangaiarkkarasi A, MeherAli R. Pattern of pathogens and their sensitivity isolated from pus culture reports in a tertiary care hospital, Puducherry Indian Journal of Basic and Applied Medical Research; December 2014: Vol.-4, Issue- 1, P. 243-248.

Ravichitra, K.N., P. Hema Prakash, S. Subbarayudu and U. Sreenivasa Rao. Isolation and antibiotic sensitivity of Klebsiella pneumonia from pus, sputum and urine samples. International journal of current microbiology and applied science. 2014; 3(3): 115-119.
Sarathbabu, R., Dr.T.V.Ramani, Dr. K.Bhaskara rao and Dr.Supriya Panda. Antibiotic susceptibility pattern of Klebsiella pneumoniae isolated from sputum, urine and pus samples. IOSR Journal of Pharmacy and Biological Sciences. 2012; 1:4-9.

Vijeta Sharma, Geeta Parihar, Vijaylaxmi Sharma, Harshita Sharma. A Study of Various Isolates from Pus Sample with Their Antibiogram from Jln Hospital, Ajmer. IOSR Journal of Dental and Medical Sciences. Volume 14, Issue 10 Ver. VI (Oct. 2015), PP 64-68.

Zubair M, Malik A, Ahmad J. Clinicomicrobiological study and antimicrobial drug resistance profile of diabetic foot infections in North India. Foot 2011 Mar; 21(1):6-14. Epub 2010 Dec 4.

\section{How to cite this article:}

Nithya Gomatheswari, S. and Jeyamurugan, T. 2017. Bacteriological Profile and the Antibiotic Susceptibility Pattern of Microorganisms Isolated from Pus/Wound Swab Isolates in Patients Attending a Tertiary Care Hospital in South India. Int.J.Curr.Microbiol.App.Sci. 6(10): 14051413. doi: https://doi.org/10.20546/ijcmas.2017.610.166 